\title{
Esthetic Improvement through Orthodontic Treatment Involving Extraction: Use of Orthodontic Anchor Screws
}

\author{
Hidenori Katada \\ Department of Orthodontics, Tokyo Dental College, \\ 2-9-18 Kanda-Misakicho, Chiyoda-ku, Tokyo 101-0061, Japan
}

Received 23 July, 2018/Accepted for Publication 31 July, 2018

Published Online in J-STAGE 10 April, 2019

\begin{abstract}
Here, we report two cases of dental bimaxillary protrusion in which orthodontic anchor screws were used to improve the esthetics of the mouth and lips and facilitate complete closure of the mouth. Case 1 was a woman with dental maxillary protrusion and constriction of the dental arches, with the main complaint of protrusion of the lips and mandibular crowding. The maxillary and mandibular dental arches were first widened and traction applied to the maxilla via orthodontic anchor screws. The maxillary incisors were retracted $10 \mathrm{~mm}$ and the mandibular incisors $3 \mathrm{~mm}$, resulting in upper lip retraction of $3 \mathrm{~mm}$ and bottom lip retraction of $5 \mathrm{~mm}$ with respect to the E-line. The nasolabial angle increased to $20^{\circ}$, and the inter-vermilion angle, which indicates the thickness of the vermillion, decreased to $20^{\circ}$, improving esthetics by reducing the thickness of the lips. Case 2 was a woman with bimaxillary dental protrusion, with the chief complaint of protruding lips and a gummy smile. Orthodontic anchor screws were used to achieve posterior traction and intrusion in the maxillary incisor region to improve the gummy smile. The maxillary incisors were retracted $9 \mathrm{~mm}$ and the mandibular incisors $8 \mathrm{~mm}$. In the soft tissues, this yielded upper lip retraction of $3 \mathrm{~mm}$ and bottom lip retraction of $4 \mathrm{~mm}$ with respect to the E-line. The nasolabial angle increased to $10^{\circ}$ and the inter-vermilion angle decreased to $30^{\circ}$, improving esthetics by reducing the thickness of the lips. The gummy smile was also improved, with $4 \mathrm{~mm}$ intrusion of the maxillary incisors. Increased ease in closing of the mouth was also a major factor in patient satisfaction with the results of orthodontic treatment.
\end{abstract}

Key words: Adult orthodontic treatment - Orthodontic anchor screw-

E-line - Nasolabial angle - Inter-vermilion angle

\section{Introduction}

Recent years have seen an increase in demand for improved esthetics as a goal of orthodontic treatment in adults. This has increasingly involved not only improvement of crowding or over-occlusion, but also that of protrusion of the lips ${ }^{16)}$. In the past, anchorage by means of intermaxillary elastics or orthodontic headgear has been required in 
cases where the therapeutic strategy called for maximal anchoring. In such cases, however, outcome has often depended on the degree of cooperation from the patient $t^{6,9)}$. These days, however, orthodontic anchor screws are available in cases of extraction requiring maximal anchoring ${ }^{5,24)}$. Anchor screws are also reported to be effective in the improvement of a gummy smile ${ }^{24)}$.

Here, we report a dramatic improvement in esthetics by means of orthodontic anchor screws in two patients who visited our clinic with the main complaint of protrusion of the lips. Improvement of the shape of the mouth can be evaluated in reference to the E-line $\mathrm{e}^{11-13)}$ or nasolabial angle ${ }^{3,10)}$. Morphological change was observed in the lips in both the present cases, so change in the vermilion was also evaluated $^{14,23)}$. In both cases, improvement of labial morphology was achieved to the satisfaction of the patient through retraction of the maxillary central incisors by over $10 \mathrm{~mm}$.

The patients provided written informed consent for publication of the results of their orthodontic treatment.

\section{Case Presentation}

\section{Case 1: Constricted dental arches and crowding with Angle Class I and maxillary protrusion}

This patient was a woman aged 31 years and 9 months who visited our clinic with the main complaint of protrusion of the lips and mandibular crowding. She had experienced the onset of temporomandibular joint pain 10 years earlier, and was currently using a splint at night. She had attended the Department of Psychosomatic Medicine at another hospital 5 years earlier for depression, for which she had been prescribed medication and was still under observation. She felt that the mandibular crowding had worsened over the previous year or two.

Her family medical history revealed protruding teeth in her father and elder brother, but healthy dentition in her mother.

The findings were a convex-type facial profile, no frontal bilateral asymmetry, tension of the mentalis muscle when the mouth was closed, and a non-gummy smile.

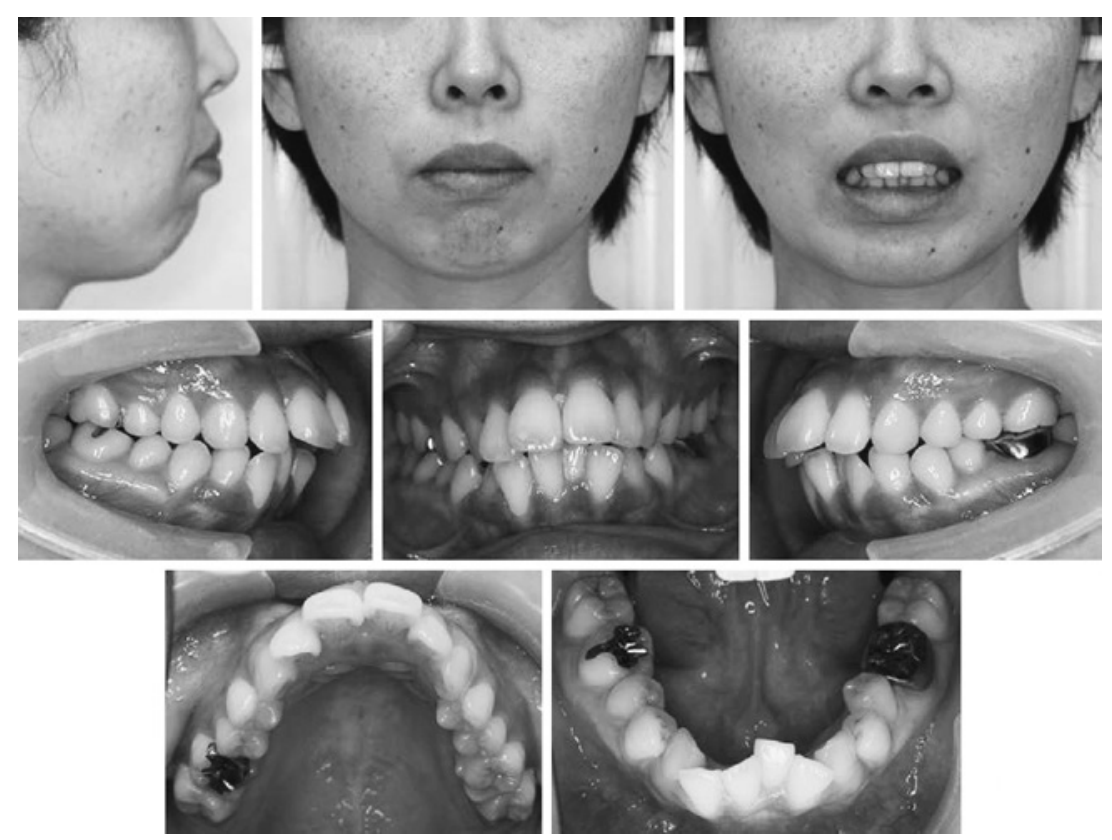

Fig. 1 Case 1: Intraoral and facial photographs at pre-treatment 
Table 1 Case 1: Measurements on pre-, post-treatment, and retention cephalometric radiographs

\begin{tabular}{|c|c|c|c|}
\hline & Pre-treatment & Post-treatment & $\begin{array}{c}\text { Retention } \\
\quad(4 \mathrm{yr})\end{array}$ \\
\hline & $31 Y 9 M$ & $35 \mathrm{Y} 5 \mathrm{M}$ & 39Y6M \\
\hline SNA (deg.) & 80.3 & 79.7 & 79.9 \\
\hline SNB (deg.) & 76.9 & 75.1 & 75.5 \\
\hline ANB (deg.) & 3.4 & 4.6 & 4.4 \\
\hline Facial angle (deg.) & 83.7 & 83.5 & 83.5 \\
\hline Y-axis (deg.) & 65.4 & 65.1 & 65.2 \\
\hline FMA (deg.) & 34.7 & 34 & 34.2 \\
\hline Occ. Plane to SN (deg.) & 23.1 & 25.5 & 25.3 \\
\hline U1 to $\mathrm{FH}$ (deg.) & 121.1 & 98.6 & 99.1 \\
\hline IMPA (L1 to MP) (deg.) & 97.4 & 97.7 & 97.6 \\
\hline FMIA (deg.) & 47.8 & 48.4 & 48.2 \\
\hline Interincisal angle (deg.) & 106.7 & 129.8 & 129.4 \\
\hline U1 to A-Pog $(\mathrm{mm})$ & 16 & 5.9 & 6.1 \\
\hline L1 to A-Pog (mm) & 6.8 & 3.8 & 4.1 \\
\hline E-line: Upper (mm) & 2 & -1 & -1 \\
\hline E-line: Lower (mm) & 4.8 & 0 & 0 \\
\hline Nasolabial angle (deg.) & 88 & 112 & 111 \\
\hline Inter-vermilion angle (deg.) & 142 & 122 & 120 \\
\hline Upper vermilion depth (mm) & 13 & 10 & 10 \\
\hline Lower vermilion depth (mm) & 13 & 7 & 6 \\
\hline Overjet $(\mathrm{mm})$ & 9 & 2.5 & 3 \\
\hline Overbite (mm) & 2 & 2 & 3 \\
\hline
\end{tabular}

Intraoral findings revealed overjet of $9 \mathrm{~mm}$, overbite of $2 \mathrm{~mm}$, and an Angle Class I molar relationship. The dental arch morphology was a V-shaped dental arch in the maxilla and a constricted dental arch in the mandible, with considerable crowding of the maxillary and mandibular incisors (Fig. 1).

With regard to the skeletal pattern, the anteroposterior positional relationship of the maxilla and mandible on the cephalometric X-ray was an SNA angle of $80^{\circ}$, SNB angle of $77^{\circ}$, and ANB angle of $3^{\circ}$, which were roughly normal values. Vertically, the FMA was high, at $35^{\circ}$. As to the dental pattern, the maxillary incisors showed considerable labial inclination and forward displacement, with a U1 to $\mathrm{FH}$ of $121^{\circ}$ and $\mathrm{U} 1$ to A-Pog of $16 \mathrm{~mm}$. The mandibular incisors showed considerable labial inclination, with an FMIA of $48^{\circ}$ and L1 to A-Pog of $7 \mathrm{~mm}$. As to the soft tissues, the facial profile was convex-type, with protrusion with respect to the E-line of $2 \mathrm{~mm}$ in the upper lip and $5 \mathrm{~mm}$ in the lower lip (Table 1, Fig. 2). The frontal facial aspect was roughly symmetrical, and the tooth relationship was roughly in agreement with the center line.

1) Clinical procedures and outcomes

Based on the above findings, the diagnosis was Angle Class I and maxillary protrusion accompanying constriction of the dental arches and crowding.

The therapeutic strategy was to extract the maxillary and mandibular first premolars, and to use a multibracket appliance to relieve crowding and move the incisors distally. It was also decided to treat the constriction of the maxillary and mandibular dental arches by enlarging them with a quad helix appliance in 
the maxilla and a bi-helix appliance in the mandible. In terms of mechanics, the excessive overjet was treated by implanting screwtype orthodontic anchors in the maxillary molar region to give maximum anchorage for traction. Screw-type anchors were selected because the molar relationship was Angle Class I and there was therefore no need for distal movement of the molars. Traction was applied, with care taken to avoid bite-raising and excessive lingual inclination. The mandibular crowding was relieved by extraction of the lower first premolar. The gap was closed taking care not to move the mandibular incisors too far in the lingual direction. The lip protrusion was improved by achieving the appropriate overjet through distal movement of the maxillary incisors.

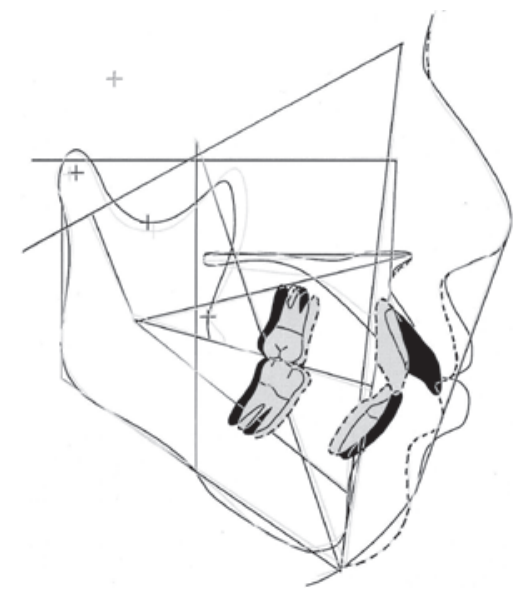

Fig. 2 Case 1: Trace superimposition before (black line) and after treatment (dashed line)
First, a quad helix was fitted to the maxilla and a bi-helix to the mandible. A multibracket appliance (Roth bracket, $0.022 \times 0.028$ inch; Sankin, Japan) was subsequently fitted to level the maxillary and mandibular dental arches while they were being widened (Fig. 3). The quad helix and bi-helix were removed after one year. A $0.019 \times 0.025$ stainless steel wire with hooks was fitted in the maxilla and traction performed using hyper-elastic coil springs with the orthodontic anchor screws as anchorage (Fig. 4). A $0.019 \times 0.025$ stainless steel wire was fitted to the mandible, after which the gap was closed by distal movement of the canines and the incisors within the mandible. Class II intermaxillary elastics were subsequently fitted to establish intercuspation. The period of active treatment was 3 years and 2 months (Fig. 5).

The treatment outcome was as follows: the values from cephalometric analysis of the upper incisors revealed that $\mathrm{U} 1$ to $\mathrm{FH}$ improved from 121 to $99^{\circ}$ and U1 to A-Pog from 16 to $6 \mathrm{~mm}$; in the lower incisors FMIA remained unchanged at $48^{\circ}$, while L1 to A-Pog moved lingually from 7 to $4 \mathrm{~mm}$. As a result, the interincisal angle increased from 107 to $130^{\circ}$. In the skeletal pattern, no major change was seen in ANB or FMA (Table 1, Fig. 2).

Panoramic X-ray findings revealed that the tooth roots had a satisfactory parallel relationship, but the upper incisor roots showed slight resorption (Fig. 6).

In the soft tissues, prior to treatment the facial profile was convex-type, with upper lip protrusion of $2 \mathrm{~mm}$ and lower lip protrusion
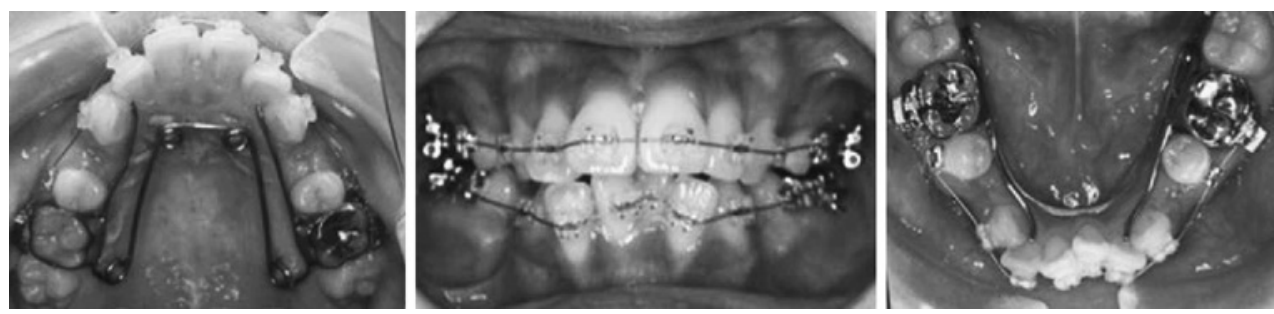

Fig. 3 Case 1: Photograph during treatment

Leveling and expansion of maxilla and mandible 

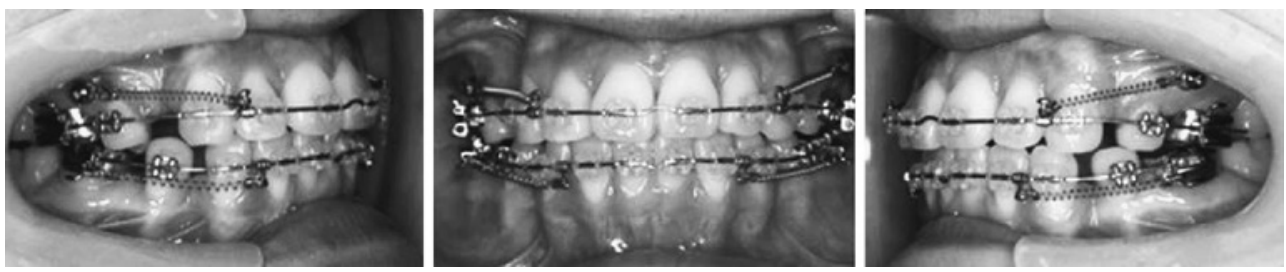

Fig. 4 Case 1: Photograph during treatment

Posterior traction from implant anchor
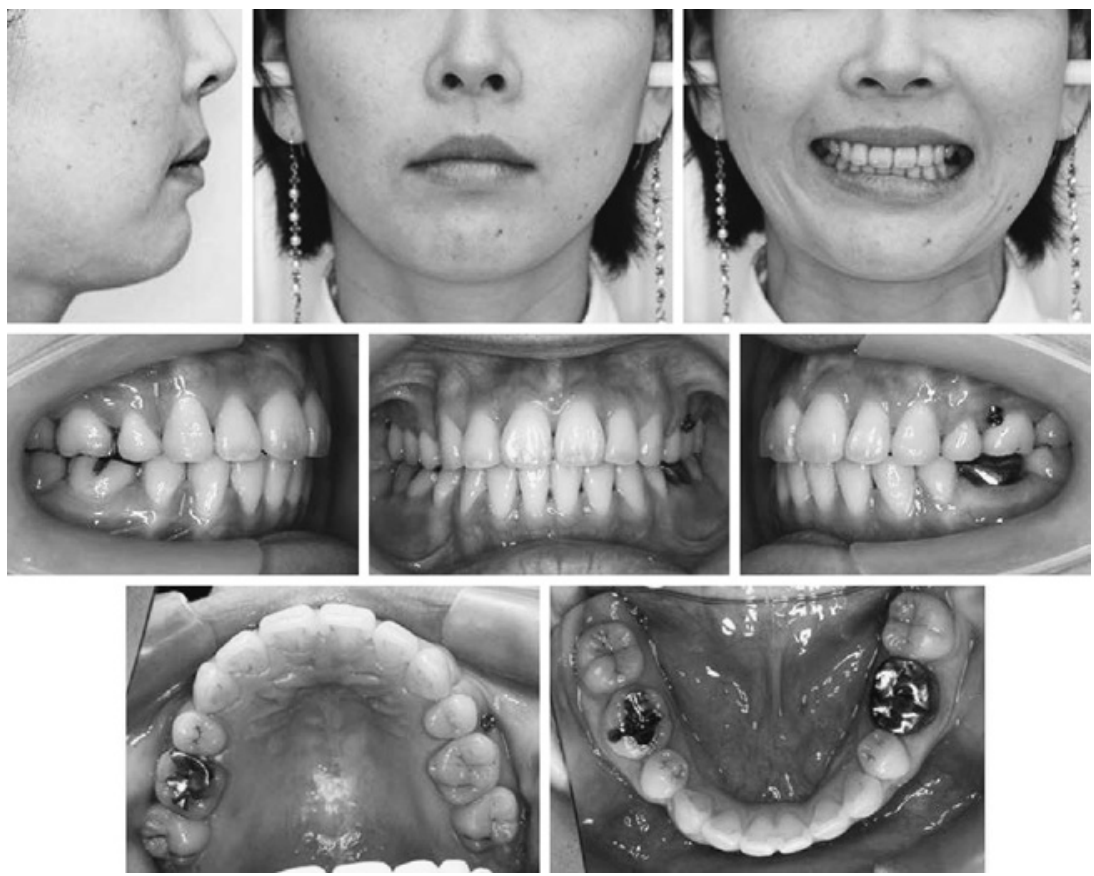

Fig. 5 Case 1: Intraoral and facial photographs at post-treatment

of $5 \mathrm{~mm}$ with respect to the E-line. There was considerable retraction as a result of the treatment, with protrusion reduced to $-1 \mathrm{~mm}$ in the upper lip and $0 \mathrm{~mm}$ in the lower lip (Fig. 7). In addition, the nasolabial angle increased from 90 to $110^{\circ}$ (Fig. 8). The inter-vermilion angle, which indicates the thickness of the vermilion, decreased from 142 to $122^{\circ}$ (Fig. 9). In addition, with the straight line passing through the alar curvature point (Ac) and perpendicular to plane $\mathrm{FH}$ taken as the Y-axis, the distance to the upper vermilion (upper vermilion depth) decreased from 13 to
$10 \mathrm{~mm}$, and the distance to the lower vermilion (lower vermilion depth) from 13 to $7 \mathrm{~mm}$ (Fig. 10). Tension of the mentalis muscle when the mouth was closed due to protrusion of the upper incisors at pre-treatment had disappeared after treatment.

A wraparound retainer was fitted to the maxilla, which the patient was instructed to wear all day for one year, after which use was reduced to only at night. The patient currently still uses the retainer at night. In the mandible, a fixed retainer was fitted from the mesial marginal ridge line of the second pre- 

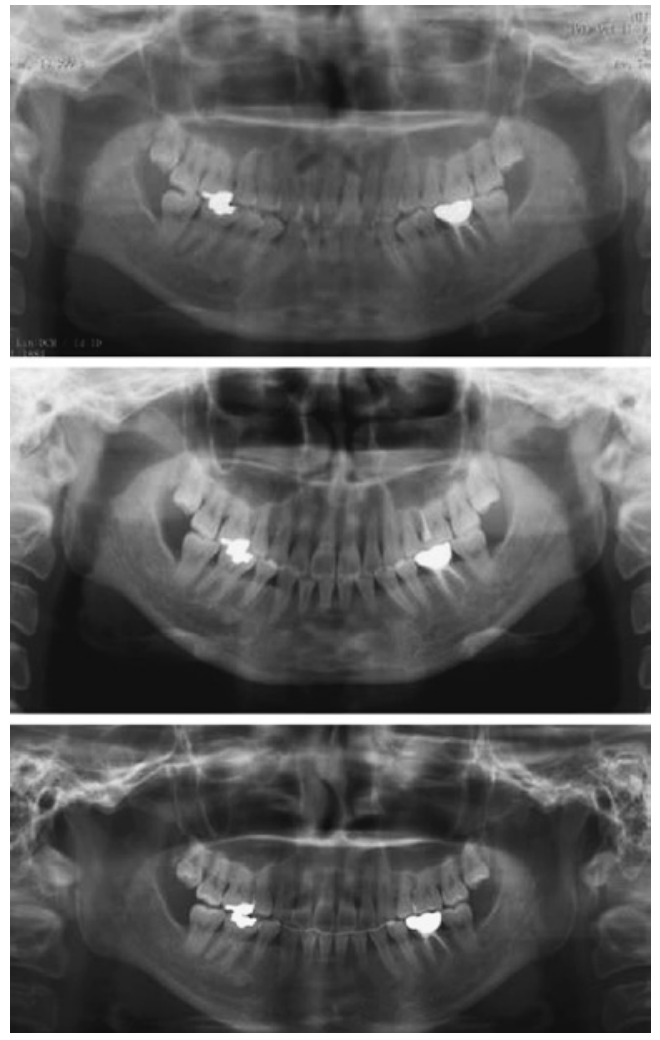

Fig. 6 Case 1: Panoramic X-ray photographs from above taken at pre-treatment, post-treatment, and 3 years post-treatment
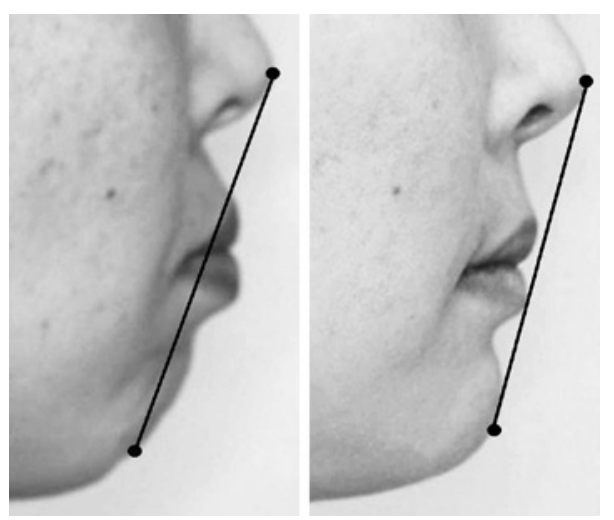

Fig. 7 Case 1: E-line photographs at pre- and post-treatment

molar to the opposing side, and after 3 years it is still being used. The frequency of use of the maxillary retainer decreased from around

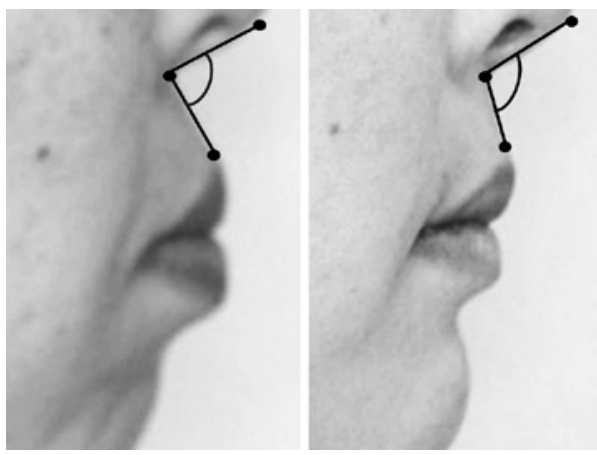

Fig. 8 Case 1: Nasolabial angle photographs at preand post-treatment

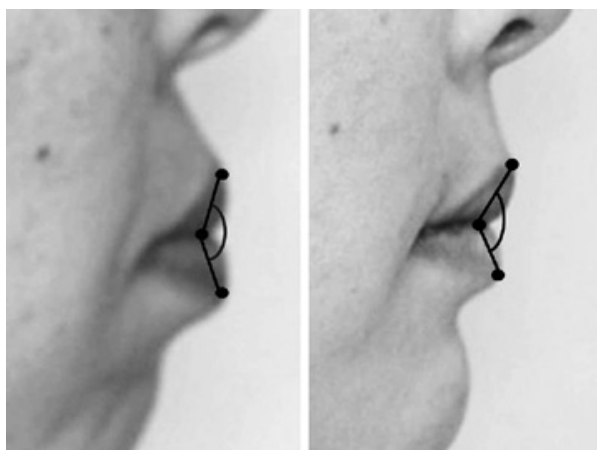

Fig. 9 Case 1: Inter-vermilion angle photographs at pre- and post-treatment

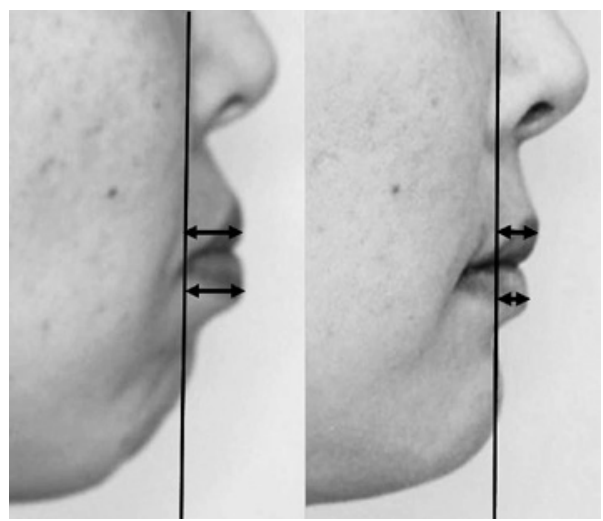

Fig. 10 Case 1: Upper and lower vermilion depth photographs at pre- and post-treatment

the second year, however, resulting in the appearance of a space in the maxillary first premolar region (Fig. 11). 


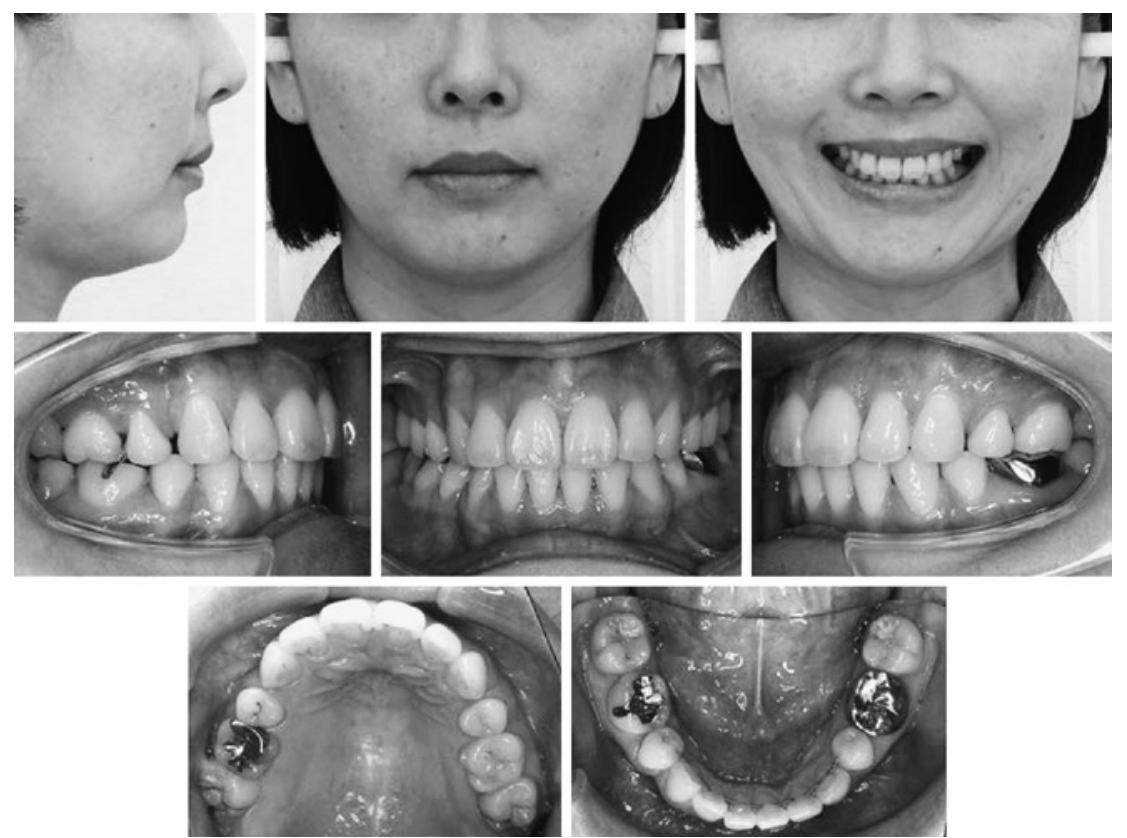

Fig. 11 Case 1: Intraoral and facial photographs at 3 years after end of active treatment

\section{Case 2: Angle Class I and bimaxillary protrusion accompanying gummy smile}

This patient was a woman aged 19 years and 7 months who visited the clinic with the main complaint of protruding lips and a gummy smile. The gummy smile had bothered her from junior high school onward, and she had been recently referred to this clinic after consultation with her uncle, an oral surgeon. Her family history revealed that both parents had healthy dentition.

The findings for this patient were a convextype facial profile, no frontal bilateral asymmetry, tension of the mentalis muscle when the mouth was closed, and a gummy smile.

Intraoral findings revealed overjet of $4 \mathrm{~mm}$, overbite of $2 \mathrm{~mm}$, and an Angle Class I molar relationship. No abnormalities were found in the dental arch morphology, and there was only slight crowding. The tooth crown diameters were slightly on the large side in both the maxilla and the mandible, but the tooth size ratio was normal (Fig. 12).

In terms of skeletal pattern, the cephalometric X-ray revealed that the anteroposterior positional relationship of the maxilla and mandible was an SNA angle of $72^{\circ}$ and an SNB angle of $70^{\circ}$, so that both showed retrusion, and the relative positional relationship was an ANB angle of $2^{\circ}$, which was a normal value. Vertically, the FMA was $31^{\circ}$, which was slightly high, but still within the normal range. In terms of dental pattern, the maxillary incisors showed considerable labial inclination and forward displacement, with a $\mathrm{U} 1$ to $\mathrm{FH}$ of $117^{\circ}$ and U1 to A-Pog of $14 \mathrm{~mm}$. The mandibular incisors also showed labial inclination, with an FMIA of $42^{\circ}$ and L1 to A-Pog of $11 \mathrm{~mm}$. As for the soft tissues, the facial profile was convex-type, with protrusion with respect to the E-line of $2 \mathrm{~mm}$ in the upper lip and $4 \mathrm{~mm}$ in the lower lip (Table 2, Fig. 13). The frontal facial aspect was roughly symmetrical, and the tooth relationship was roughly in agreement with the center line.

1) Clinical procedures and outcomes

Based on the above findings, the diagnosis was Angle Class I and bimaxillary protrusion accompanying a gummy smile.

The therapeutic strategy was to extract the 


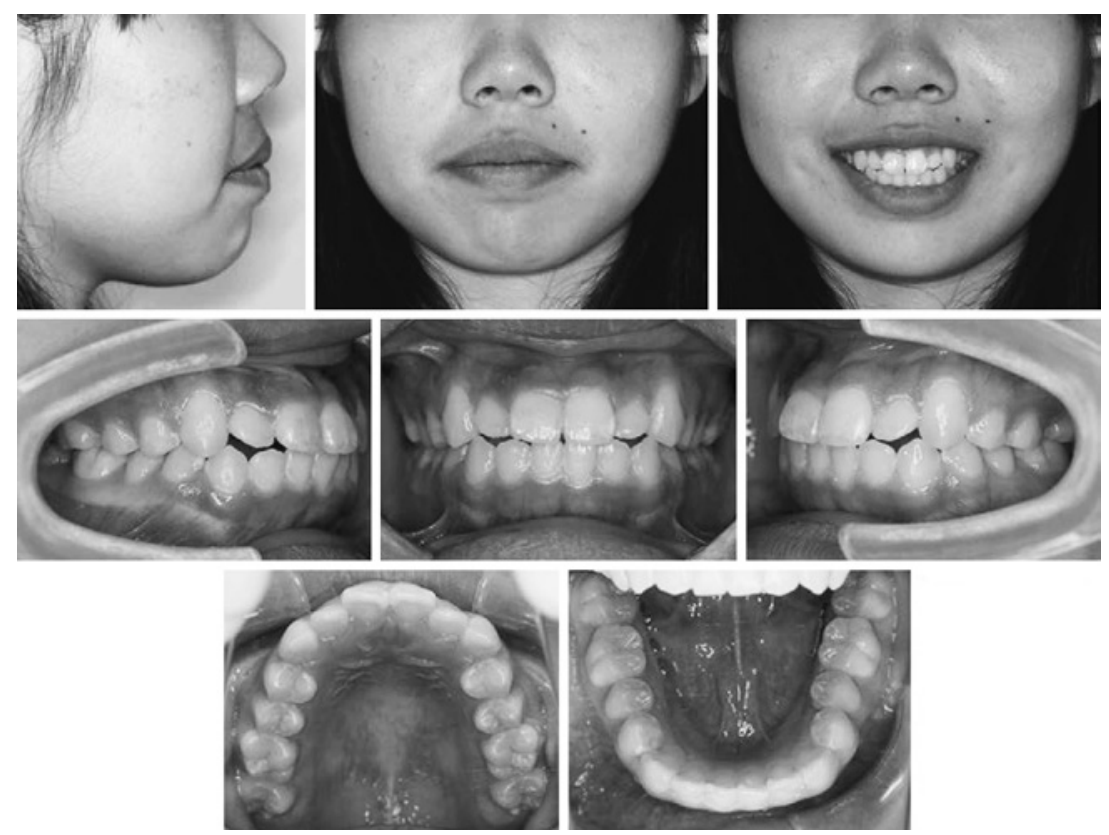

Fig. 12 Case 2: Intraoral and facial photographs at pre-treatment

maxillary and mandibular first premolars and use a multibracket appliance for distal movement of the maxillary and mandibular incisors. In terms of mechanics, orthodontic anchor screws were implanted in the maxillary and mandibular molar regions to give maximum anchorage for traction. Screw-type anchors were selected because the molar relationship was Angle Class I and there was therefore no need for distal movement of the molars. The lip protrusion was improved through distal movement of the maxillary and mandibular incisors. In addition, the gummy smile was addressed by implanting orthodontic anchor screws in the maxillary incisor alveolar region to intrude the upper incisors.

Following extraction of the maxillary and mandibular first premolars, maxillary and mandibular multibracket appliances (Roth bracket, $0.022 \times 0.028$ inch; Sankin, Japan) were fitted and leveling of the dental arch carried out using copper Ni-Ti wire. Orthodontic anchor screws were implanted in the maxillary and mandibular first molar mesial region and maxillary canine mesial alveolar region. After 5 months, $0.019 \times 0.025$ stainless steel wires with hooks were fitted to the maxilla and mandible and traction commenced by means of hyper-elastic coil springs, with the orthodontic anchor screws providing anchorage. At one year following commencement of traction, the multibracket appliances were temporarily removed, leaving the first molar bands and orthodontic anchor screws, as the patient was going to study abroad. The patient had an invisible retainer to use while abroad, but rarely wore it.

Treatment was recommenced at 1 year and 4 months later when the patient returned to Japan. Leveling was carried out using copper $\mathrm{Ni}$-Ti wires, and after 4 months $0.019 \times 0.025$ stainless steel wires were fitted and traction commenced using the orthodontic anchor screws as anchorage. The incisors were pulled upward by elastics. Vertical elastics were used in the maxillary and mandibular incisor regions at night to prevent development of open bite (Fig. 14).

The period of active treatment was 4 years 
Table 2 Case 2: Measurements on pre-, post-treatment, and retention cephalometric radiographs

\begin{tabular}{|c|c|c|c|}
\hline & Pre-treatment & Post-treatment & $\begin{array}{c}\text { Retention } \\
\quad(3 \mathrm{yr})\end{array}$ \\
\hline & $19 Y 9 M$ & $25 \mathrm{Y} 3 \mathrm{M}$ & 28Y4M \\
\hline SNA (deg.) & 72.1 & 72.4 & 72.3 \\
\hline SNB (deg.) & 70.4 & 71.1 & 70.9 \\
\hline ANB (deg.) & 1.7 & 1.3 & 1.4 \\
\hline Facial angle (deg.) & 83.9 & 84.4 & 84.3 \\
\hline Y-axis (deg.) & 63 & 62.7 & 62.7 \\
\hline FMA (deg.) & 30.8 & 30.4 & 30.5 \\
\hline Occ. Plane to SN (deg.) & 30.2 & 20.7 & 20.9 \\
\hline U1 to FH (deg.) & 116.5 & 102.8 & 103.3 \\
\hline IMPA (L1 to MP) (deg.) & 107.7 & 81.9 & 82.2 \\
\hline FMIA (deg.) & 41.5 & 67.8 & 67.3 \\
\hline Interincisal angle (deg.) & 105 & 145 & 144.2 \\
\hline U1 to A-Pog $(\mathrm{mm})$ & 14.1 & 5.1 & 5.3 \\
\hline L1 to A-Pog (mm) & 10.7 & 3.2 & 3.3 \\
\hline E-line: Upper (mm) & 2 & -1 & -1 \\
\hline E-line: Lower (mm) & 4 & 0 & 0 \\
\hline Nasolabial angle (deg.) & 97 & 108 & 110 \\
\hline Inter-vermilion angle (deg.) & 140 & 110 & 113 \\
\hline Upper vermilion depth (mm) & 20 & 10 & 10 \\
\hline Lower vermilion depth (mm) & 20 & 5 & 6 \\
\hline Overjet $(\mathrm{mm})$ & 2.5 & 2.5 & 2.5 \\
\hline Overbite (mm) & 1.5 & 2.5 & 2.5 \\
\hline
\end{tabular}

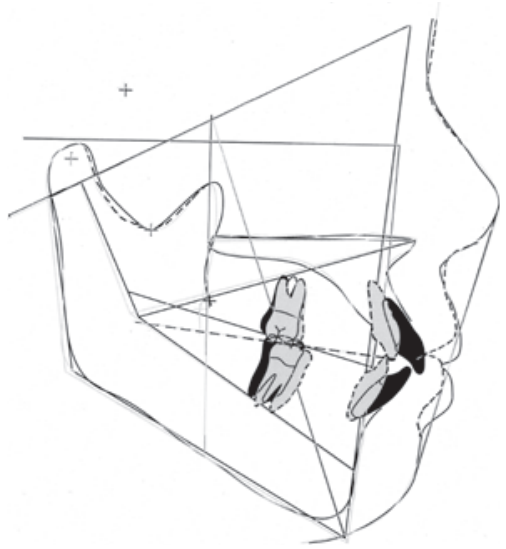

Fig. 13 Case 2: Trace superimposition before (black line) and after treatment (dashed line) and 10 months, including the period spent studying abroad. During this time, the appliances were removed and treatment suspended for a period of 1 year and 4 months (Fig. 15).

The treatment outcome was as follows: cephalometric analysis of the maxillary incisors revealed that $\mathrm{U} 1$ to $\mathrm{FH}$ improved from 117 to $103^{\circ}$ and U1 to A-Pog from 14 to $5 \mathrm{~mm}$. In the mandibular incisors, the FMIA improved from 42 to $68^{\circ}$ and L1 to A-Pog from 11 to $3 \mathrm{~mm}$. As a result, the interincisal angle increased from 105 to $145^{\circ}$. No major change was seen in any aspect of the skeletal pattern (Table 2, Fig. 13).

Panoramic X-ray findings revealed that the tooth roots had a satisfactory parallel relationship, but the upper incisor roots showed resorption (Fig. 16).

In the soft tissues, prior to treatment the 

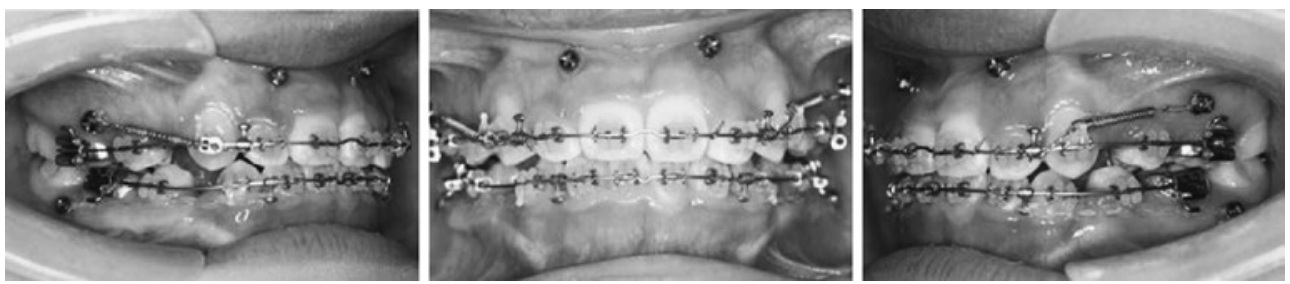

Fig. 14 Case 2: Photograph during treatment

Posterior traction from implant anchor and upward traction of maxillary incisors
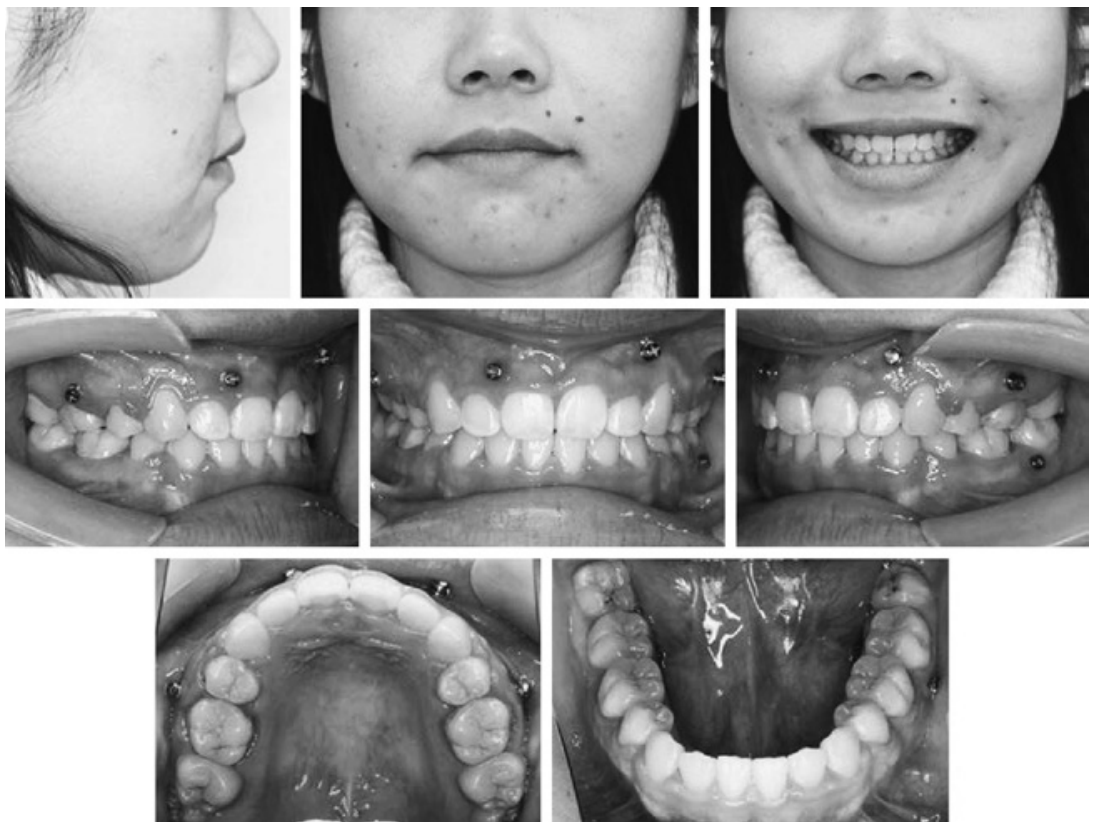

Fig. 15 Case 2: Intraoral and facial photographs at post-treatment

facial profile was convex-type, with upper lip protrusion of $2 \mathrm{~mm}$ and lower lip protrusion of $4 \mathrm{~mm}$ with respect to the E-line. Treatment resulted in considerable retraction, with protrusion reduced to $-1 \mathrm{~mm}$ in the upper lip and $0 \mathrm{~mm}$ in the lower lip (Fig. 17). In addition, the nasolabial angle increased from 100 to $110^{\circ}$ (Fig. 18). The inter-vermilion angle decreased from 140 to $110^{\circ}$ (Fig. 19). In the distance from the Y-axis to the vermilion as well, the depth of the upper vermilion decreased from 20 to $10 \mathrm{~mm}$, and that of the lower vermilion from 20 to $5 \mathrm{~mm}$ (Fig. 20).

With regard to the gummy smile, the maxil- lary incisors showed intrusion of $4 \mathrm{~mm}$. Tension of the mentalis muscle when the mouth was closed due to protrusion of the upper incisors at pre-treatment had disappeared after treatment.

A wraparound retainer was fitted to the maxilla, and a fixed retainer from the mesial marginal ridge line of the second premolar to the opposing side in the mandible. The patient was instructed to wear these all day for one year, after which use was reduced to only at night. The patient still continues with nighttime use of these retainers (Fig. 21). 

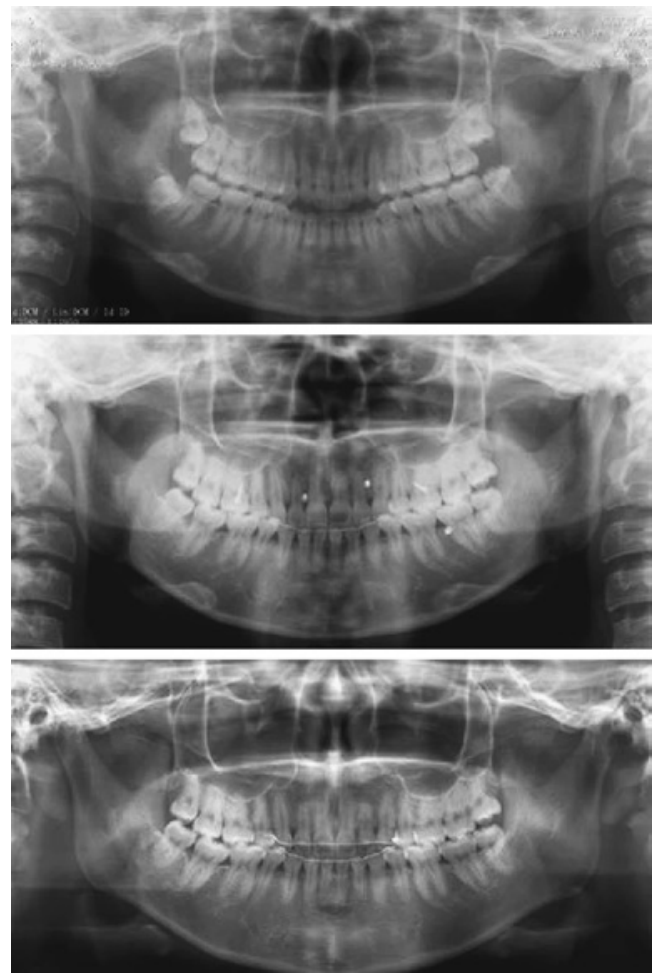

Fig. 16 Case 2: Panoramic X-ray photographs from above taken at pre-treatment, post-treatment, and 3 years post-treatment
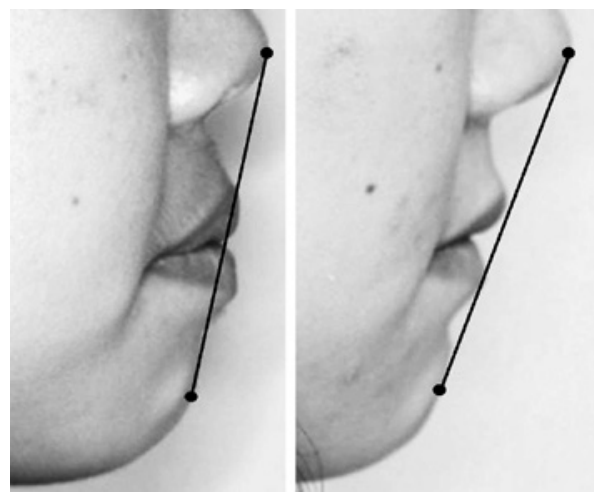

Fig. 17 Case 2: E-line photographs at pre- and post-treatment

\section{Discussion}

\section{Cases}

In recent years, there has been an increase in the number of adults receiving orthodontic

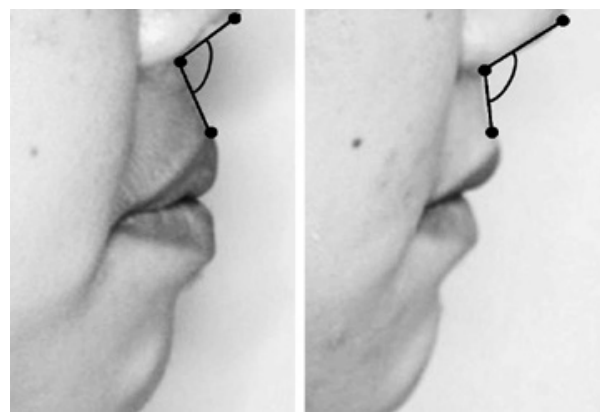

Fig. 18 Case 2: Nasolabial angle photographs at preand post-treatment

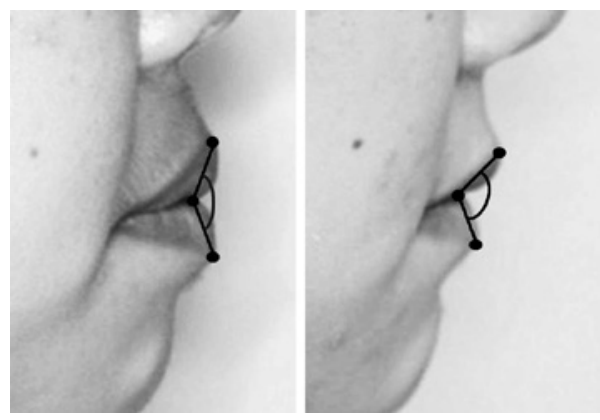

Fig. 19 Case 2: Inter-vermilion angle photographs at pre- and post-treatment
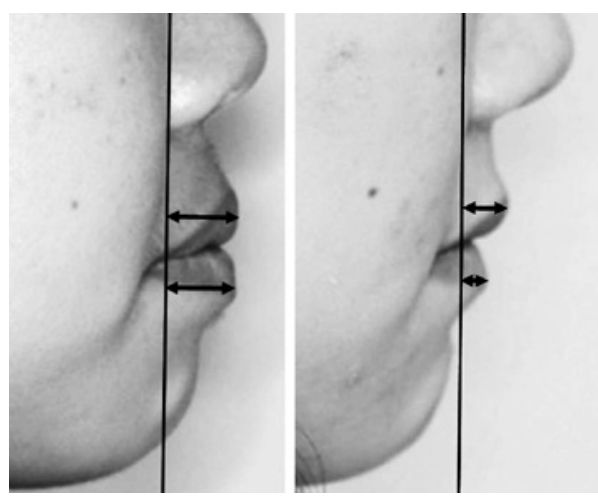

Fig. 20 Case 2: Upper and lower vermilion depth photographs at pre- and post-treatment

treatment. Adult orthodontic treatment is characterized by esthetic demands, problems regarding the number of teeth or periodontal disease, and social circumstances, and it therefore calls for different considerations 


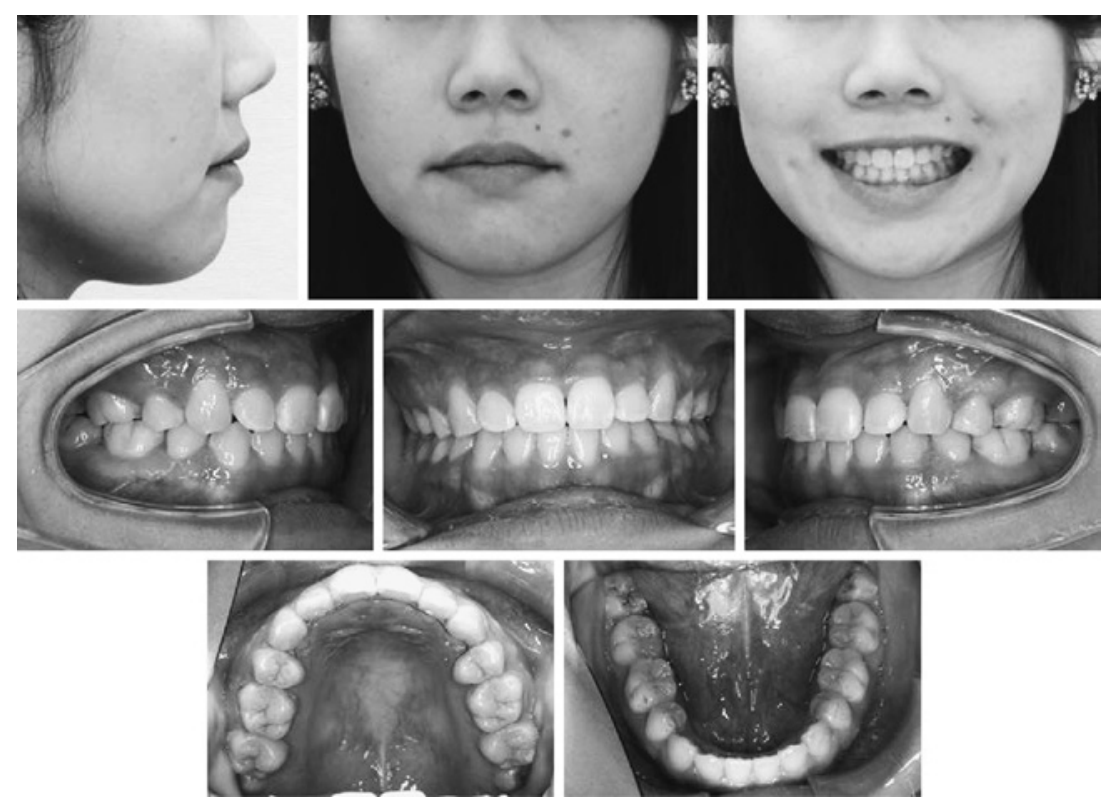

Fig. 21 Case 2: Intraoral and facial photographs at 3 years after end of active treatment

from orthodontic treatment carried out during the growth period ${ }^{16)}$. In the present cases, also, there were considerable esthetic demands to be met, and in both patients the main complaint was protrusion and incomplete closure of the lips.

Case 1 was a patient with constricted dental arches and crowding with Angle Class I and maxillary protrusion. The constricted dental arches were treated by arch enlargement with quad and bi-helix appliances. Dental arch enlargement ensured distal displacement of the incisors, on top of which the maxillary and mandibular first premolars were extracted and the incisor region displaced posteriorly. Orthodontic anchor screws were implanted in the maxilla to obtain maximum anchorage $^{24)}$. This resulted in $10 \mathrm{~mm}$ of distal movement in the maxillary incisors and $3 \mathrm{~mm}$ in the mandibular incisors. This yielded $3 \mathrm{~mm}$ of retraction in the upper lip and $5 \mathrm{~mm}$ in the lower lip with respect to the E-line, improving the facial profile from one that was convex to one that was straight. In addition, a $20^{\circ}$ increase in the nasolabial angle and $20^{\circ}$ decrease in the inter-vermilion angle improved the shape of the mouth ${ }^{10,14}$. Moreover, the patient had complained of incomplete closure of the lips due to maxillary incisor protrusion, and this also showed an improvement after treatment. Retention was used fully for 1 year, after which compliance was poor. As a result, although occlusion was stable, a gap was produced at the maxillary extraction site.

Case 2 was a patient with alveolar maxillary and mandibular protrusion, with no skeletal problems. Treatment to improve the labial inclination of the maxillary and mandibular incisors was carried out following extraction of the maxillary and mandibular first premolars. The problem in this particular case was how to control torque and intrusion with distal displacement of the maxillary incisors. Orthodontic anchor screws have been reported to be effective in improving a gummy smile $^{24)}$. In Case 2, therefore, the gummy smile was improved by applying intrusion force from orthodontic anchor screws in the maxillary incisor region at the time of distal displacement. Furthermore, a $0.019 \times 0.025$ stainless steel wire configured with a curve of 
Spee was used to slowly retract the 6 maxillary front teeth by springs from the orthodontic anchor screws, which allowed maximum anchorage and an appropriate inclination of the axes of the maxillary front teeth. At the same time, labial inclination in the mandibular front teeth was improved as well by extraction of the first premolars and traction with maximum anchorage from the orthodontic anchor screws. This resulted in distal displacement of the maxillary front teeth by $9 \mathrm{~mm}$ and the mandibular front teeth by $8 \mathrm{~mm}$. This gave $3 \mathrm{~mm}$ retraction of the upper lip and $4 \mathrm{~mm}$ retraction of the lower lip with respect to the E-line, improving the facial profile from convex to straight. The morphology of the mouth also showed an improvement, with a $10^{\circ}$ increase in the nasolabial angle and $30^{\circ}$ decrease in the inter-vermilion angle ${ }^{10,14)}$. In addition, the maxillary front teeth showed intrusion of $4 \mathrm{~mm}$, resulting in an improvement in the gummy smile ${ }^{24}$. Tooth root resorption was found in the maxillary front tooth region, however. Possible reasons for this include the effect of intrusion due to traction from the anchor screws ${ }^{15,20)}$; the extended period of time for which the appliance was fitted, which was 3 years and 6 months because use of the appliance was interrupted for 1 year and 4 months during treatment; the effect of extraction and orthodontic treatment $^{19)}$; and age-related factors in adult orthodontic treatment ${ }^{7,15)}$.

\section{Esthetic evaluation}

\section{1) E-line}

The Visual Treatment Objective (VTO) has long been used in treatment planning, with L1 to A-pog set at $3 \mathrm{~mm}$. In addition, retraction of the upper lip accompanying posterior displacement of the maxillary central incisors is believed to require $2 / 3$ the amount of movement of the central incisors ${ }^{17}$. With the lower lips, the VTO is drawn up by matching the center of the $\mathrm{OB}$ and $\mathrm{OJ}$ so that the amount of lip retraction is not directly related to the amount of posterior displacement of the mandibular incisors ${ }^{18}$. In the present study, L1 to A-pog improved from 7 to $4 \mathrm{~mm}$ in Case 1 and from 11 to $3 \mathrm{~mm}$ in Case 2, with the improved values corresponding to the standard values for Japanese people ${ }^{11)}$. One study using the facial profile in silhouette for evaluation reported that a lower lip profile of $-2 \mathrm{~mm}$ with respect to the E-line was desirable in Japanese people ${ }^{12}$. At the same time, another study basing its evaluations on silhouettes reported that the desirable profile in Japanese people was $-3.45 \mathrm{~mm}$ with respect to the $\mathrm{E}-$-line $^{13)}$. Therefore, we believe that near ideal values were obtained in both the present cases.

2) Nasolabial angle

The nasolabial angle is the angle formed by the base of the nose and the lips, which has been reported to be ideally $102.2 \pm 8^{\circ}$ in Caucasian men and $102.4 \pm 8^{\circ}$ in Caucasian women $^{8}$. Meanwhile, angles of $102.7 \pm 11^{\circ}$ have been reported in young Asian men and $101.6 \pm 11^{\circ}$ in young Asian women ${ }^{25)}$, so there are some differences between races ${ }^{1)}$. If the incisors are moved $5 \mathrm{~mm}$ posteriorly due to orthodontic treatment, then it may be estimated that the lips will move back by approximately $70 \%$ of this value, or $3.5 \mathrm{~mm}^{18)}$. Analyzing what will happen to the E-line and nasolabial angle due to change in the position of the lips with movement of the incisors in orthodontic treatment should allow better prediction of the mouth shape that will ensue. Orthodontic treatment involving extraction is effective in cases of protrusion of the lips and a small nasolabial angle ${ }^{10)}$. The nasolabial angle plays an important role in human esthetics. Clinicians should therefore place great emphasis on evaluating this area and planning a treatment mechanism that will place this angle within the acceptable standard deviation ${ }^{3)}$. Retraction of the maxillary incisors by $1 \mathrm{~mm}$ is reported to increase the nasolabial angle by $1-3^{\circ 4,21,22}$. In the present study, retraction of the maxillary incisors by $10 \mathrm{~mm}$ in Case 1 and $9 \mathrm{~mm}$ in Case 2 increased the nasolabial angle by $10^{\circ}$ in both cases, thereby improving the mouth shape in each patient.

3) Change in vermilion morphology

Another change seen in the present cases 
accompanying retraction of the lips was change in the morphology of the vermilion. One study reported a decrease in the height of the vermilion and an improvement in its morphology due to premolar extraction in patients with bimaxillary protrusion ${ }^{23)}$. Another study reported that the surface area of the upper vermilion was proportionally greater in maxillary protrusion patients, and that an increase in surface area of the lower vermilion after orthodontic treatment improved the balance between the upper and lower vermilion ${ }^{2}$. In another earlier study, which investigated differences in the morphology of the vermilion between before and after orthodontic treatment for bimaxillary protrusion, it was shown that, while there was a high proportion of inward rotational change in the upper vermilion, the lower vermilion was characterized more by parallel rather than rotational displacement ${ }^{14}$. In the present study, the inter-vermilion angle decreased from 142 to $122^{\circ}$ in Case 1 and from 140 to $110^{\circ}$ in Case 2, indicating a reduction in the thickness of the vermilion in both cases. The distance from the Y-axis to the vermilion also showed considerable retraction. In Case 1, the depth of the upper vermilion decreased from 13 to $10 \mathrm{~mm}$ and that of the lower vermilion from 13 to $7 \mathrm{~mm}$; while in Case 2, the depth of the upper vermilion decreased from 20 to $10 \mathrm{~mm}$ and that of the lower vermilion from 20 to $5 \mathrm{~mm}$.

These results indicate that esthetic improvement was obtained through morphological change in the vermilion.

\section{Conclusion (s)}

Here, extraction using orthodontic anchor screws yielded an improvement in the facial profile in adult patients with bimaxillary protrusion. The use of orthodontic anchor screws allowed control over the anchorage and appropriate distal movement of the incisors. Moreover, intrusion of the maxillary incisors allowed improvement of a gummy smile. Retraction of the upper and lower lips and morphological change in the vermilion resulted in considerable improvement in esthetics. In addition to which, increased ease in closing of the mouth was also a major factor in the patients' satisfaction with their orthodontic treatment.

\section{Conflicts of Interest}

The authors wish to declare no conflict of interest with regard to this report.

\section{Acknowledgements}

The authors wish to thank Professor Jeremy Williams of the Department of International Medical Communications at Tokyo Medical University for his assistance with the English of this manuscript.

\section{References}

1) Fradeani M (2005) Oral Imprant Rehabilitation Series, pp.27-31, Quintessence, Tokyo. (in Japanese)

2) Islam R, Kitahara $T$, Naher L, Hara A, Nakashima A (2009) Lip morphological changes in orthodontic treatment. Angle Orthod 79:256-264.

3) Jeelani W, Fida M, Shaikh A (2016) Facial soft tissue analysis among various vertical facial patterns. J Ayub Med Coll Abbottabad 28: 29-34.

4) Kachiwala VA, Kalha AS, Machado G (2009) Soft tissue changes associated with first premolar extractions in adult females. Aust Orthod J 25:24-29.

5) Kanomi R (1997) Mini-implant for orthodontic anchorage. J Clin Orthod 31:763-767.

6) Kuroda S, Yamada K, Deguchi T, Kyung HM, Takano-Yamamoto T (2009) Class II malocclusion treated with miniscrew anchorage: Comparison with traditional orthodontic mechanics outcomes. AmJ Orthod Dentofacial Orthop 135:302-309.

7) Matsuda Y, Motokawa M, Kaku M, Kawata T, Yamamoto R, Tsuka N, Inubushi T, Sasamoto T, Ozaki N, Koseki H, Kawazoe A, Abedini S, Tanne K (2011) Clinical survey of the associa- 
tion between root resorption incident to orthodontic treatment and host factors. Orthod Waves-Jpn Ed 70:21-31. (in Japanese)

8) McNamara JA, Brust EW, Riolo ML (1992) Soft tissue evaluation of individuals with an ideal occlusion and well balanced face, Esthetics and the treatment of facial form, Craniofacial growth series, McNamara JA Jr ed., pp.115-146, Center for Human Growth and Development, The University of Michigan, Ann Arbor.

9) Murase C, Nojima K, Nishii Y, Takaki T, Sueishi K (2014) Orthodontic treatment outcomes in adults with Class II div.1 extraction treated using the anchor screw-Comparison with cervical headgear and Nance holding arch-. Shikwa Gakuho 114:431-437. (in Japanese)

10) Nandini S, Prashanth CS, Somiah SK, Reddy SR (2011) An evaluation of nasolabial angle and the relative inclinations of the nose and upper lip. J Contemp Dent Pract 12:152-157.

11) Nezu H, Nagata K, Yoshida Y, Kosaka H, Kikuchi M (1982) Cephalometric comparison of clinical norms between the Japanese and Caucasians. Nihon Kyosei Shika Gakkai Kaishi 41:450-465. (in Japanese)

12) Noda S, Nodai E, Yoshimatsu F, Kusumoto O, Fujita K, Yamada K (1993) Evaluation of the relationships between the E-line and the position of the lower lip in convex, straight and concave faces. Kyushu Dental Society 47:377384. (in Japanese)

13) Nomura M, Motegi E, Yamaguchi H, Hatch JP, Rugh JD (2007) Esthetic judgement of profiles by Europian American, Hispanic, Japanese and African. Shikwa Gakuho 107: 69-75. (in Japanese)

14) Oomori Y, Nihara J, Takahashi K, Morita S, Saito I (2016) Changes in vermilion lip morphology after premolars extraction treatment in Japanese patients with bimaxillary protrusion. Orthod Waves-Jpn Ed 75:97-105. (in Japanese)

15) Parker RJ, Harris EF (1998) Directions of orthodontic tooth movements associated with external apical root resorption of the maxillary central incisor. Am J Orthod Dentofacial Orthop 114:677-683.

16) Proffit WR, Fields RP Jr (2000) Contemporary Orthodontics, 3rd ed., pp.649-659, Mosby, St.
Louis.

17) Ricketts RM (1957) Planning treatment on the basis of the facial pattern and an estimate of its growth. Angle Orthod 27:14-37.

18) Ricketts RM (1968) Esthetics, environment, and the law of lip relation. Am J Orthod 54: 272-289.

19) Sameshima GT, Sinclair PM (2001) Predicting and preventing root resorption: part II. Treatment factors. Am J Orthod Dentofacial Orthop 119:511-515.

20) Sasamoto T, Motokawa M, Matsuda Y, Kaku M, Kawada T, Ozaki N, Terao A, Tanne K (2011) A clinical survey for risk factor of root resorption incident to orthodontic treatment. The Journal of Hiroshima University Dental Society 43:113-123. (in Japanese)

21) Sundareswaran S, Vijayan R (2017) Profile changes following orthodontic treatment of class I bimaxillary protrusion in adult patients of Dravidian ethnicity: A prospective study. Indian J Dent Res 28:530-537.

22) Talass MF, Talass L, Baker RC (1987) Softtissue profile changes resulting from retraction of maxillary incisors. Am J Orthod Dentofacial Orthop 91:385-394.

23) Trisnawaty N, Ioi H, Kitahara T, Suzuki A, Takahashi I (2013) Effects of four premolar extractions on vermilion height and lip area during a posed smile in patients with bimaxillary protrusion. Aust Orthod J 29:176-183.

24) Yamamoto $T$ (2014) Present status and prospects of orthodontic treatment with miniimplants as absolute anchorage. Nihon Zessoku Kyosei Shika Gakkai Kaishi 24:31-42. (in Japanese)

25) Yuen SW, Hiranaka DK (1989) A photographic study of the facial profile of southern Chinese adolescents. Quintessence Int 20:665-676.

\section{Correspondence:}

Dr. Hidenori Katada

Department of Orthodontics,

Tokyo Dental College,

2-9-18 Kanda-Misakicho, Chiyoda-ku,

Tokyo 101-0061, Japan

Tel: $+81-3-5275-1724$

Fax: + 81-3-3262-3420

E-mail: katada@tdc.ac.jp 